\title{
MICROCLIMA, IDADES DAS MATRIZES E TEMPOS DE ESTOCAGEM INFLUEN CIAM NA PERDA DE ÁGUA DOS OVOS NO TRANSPORTE?
}

Doi:http://dx.doi.org/10.1590/1809-4430-Eng.Agric.v35n4p 635-645/2015

\section{AÉRICA C. NAZARENO ${ }^{1}$, IRAN J. O. DA SILVA ${ }^{2}$, AFRÂNIO M. C. VIEIRA ${ }^{3}$, FREDERICO M. C. VIEIRA ${ }^{4}$}

\begin{abstract}
RESUMO: Objetivou-se, por meio desta pesquisa, avaliar a perda de água de ovos férteis oriundos de diferentes idades de matrizes de corte e tempos de estocagem, durante o transporte. A pesquisa foi conduzida em uma empresa integradora avícola no Estado de São Paulo, por meio do acompanhamento de três carregamentos em diferentes estações do ano. Foram utilizadas diferentes densidades de caixas durante as três viagens. Porém, para o estudo da perda de água dos ovos, foi usado um total de 18 caixas contendo 4.320 ovos, em um caminhão climatizado. Estas caixas de ovos eram referentes às três idades das matrizes e aos três intervalos de tempos de estocagem; portanto, em cada viagem, foram transportadas seis caixas com 1.440 ovos. A caracterização microclimática do contêiner foi realizada por meio de 18 dataloggers no interior das caixas, os quais registraram temperatura do ar, umidade relativa e entalpia específica. A avaliação da perda de água dos ovos foi realizada através da massa de 140 ovos individualizados de cada tratamento. $\mathrm{O}$ delineamento experimental utilizado foi o inteiramente aleatorizado, com esquema fatorial $3 \times 3$. As condições microclimáticas das cargas transportadas para todas as estações estiveram acima das faixas ideais. Independentemente da idade da matriz e da condição do microclima, o aumento do tempo de estocagem leva a maior perda de água, assim como a piores condições microclimáticas.
\end{abstract}

PALAVRAS-CHAVE: ambiência; operação pré-porteira; avicultura; perda de massa; ovos férteis.

\section{MICROCLIMATE, AGES OF BREEDERS AND STORAGE TIMES INFLUENCE ON WATER LOSS OF EGGS ON TRANSPOTATION?}

\begin{abstract}
The objective of this research was to evaluate the influence of the storage period and breeder age on water loss during fertile eggs transport. The research was conducted in a poultry integrator company in the State of São Paulo - Brazil. Three shipments were evaluated in different seasons and box density. The study of egg water loss used a total of 18 boxes containing 4,320 eggs in acclimatized truck six boxes of each treatment that consisted of three storage periods - totalizing 1,400 eggs - per shipment. The microclimate characterization of the container was evaluated by 18 dataloggers inside the boxes, which recorded the following variables: dry-bulb temperature, relative humidity, and enthalpy. The evaluation of water loss eggs was performed by mass of 140 eggs of each individual treatment. The experimental design used was completely randomized with factorial scheme $3 \times 3$. The microclimate conditions of cargo transported for all seasons were above the upper optimum range. Regardless of breeder age and microclimate conditions, increased storage time leads to greater water loss, and worst microclimate conditions.
\end{abstract}

KEYWORDS: ambience; pre-gate operation; poultry; weight loss; fertile eggs.

\footnotetext{
${ }^{1}$ Eng $^{\circ}$ A grônoma, Pós-doutorado, NUPEA - Departamento de Engenharia Biossitemas, ESALQ/USP. Piracicaba - SP, Fone: (19) 3447-8563, aericacn@y ahoo.com.br

${ }^{2}$ Eng $^{\circ}$ A grícola, Prof. Associado, NUPEA - Departamento de Engenharia Biossitemas, ESALQ/USP. Piracic aba - SP, iranoliveira@usp.br

${ }^{3}$ Estatístico, Prof. Adjunto, Departamento de Estatística, UFSCar. São Carlos - SP, afranio@ufscar.br

${ }^{4}$ Zootecnista, Prof. Adjunto, GEBIOMET - Grupo de Estudos em Biometeorologia, UTFPR. Dois Vizinhos - PR, fredericovieira@utfpr.edu.br 


\section{INTRODUÇÃO}

Os componentes dos ovos férteis, juntamente com o embrião podem ser prejudicados quando as condições de transporte, microclima e o nível de vibração são combinadas inadequadamente (ANDERSON et al., 2008; OVIEDO-RONDÓN et al., 2009, NAZARENO et al., 2013).

Nesta situação, a interação do microclima dentro do caminhão contêiner com a fisiologia do ovo torna-se um desafio, devido à grande variabilidade térmica nas diferentes regiões brasileiras. Posteriormente, a produção de pintos de corte pode ser afetada devido ao mau condicionamento de ovos durante o transporte. Em função das necessidades de se manter o controle das condições térmicas entre 10 e $20^{\circ} \mathrm{C}$ e umidade relativa de 55 a $75 \%$, faz-se necessário maior controle térmico na carroceria de transporte, para evitar a condensação de água na casca dos ovos (FASENKO et al., 1991; AKYUREK \& OKUR, 2009; SALAHI et al., 2012).

Durante o transporte dos ovos férteis, pode ocorrer redução de massa por conta da perda de água e do dióxido de carbono $\left(\mathrm{CO}_{2}\right)$. À medida que isso acontece, o espaço da câmara de ar aumenta de tamanho, enquanto as propriedades físico-químicas do albúmen mudam, tornando-o mais claro, menos viscoso e com um pH mais elevado (GAST \& HOLT, 2000; ANDERSON et al., 2008; SALAHI et al., 2012). Esta perda de água pode ser reduzida quando as condições microclimáticas do caminhão contêiner se encontram dentro das faixas ideais (DAMRON et al., 1994).

Outro fator que pode influenciar na perda de água dos ovos é a idade da matriz, pois, com o seu envelhecimento, aumenta-se a capacidade da casca do ovo em permitir trocas gasosas entre o embrião e o ambiente (MADEDDU et al., 2013). Essa habilidade está relacionada com o número, as dimensões dos poros e com a espessura e a resistência da casca (TANURE et al., 2009; NAZARENO et al., 2014a).

Desta forma, faz-se necessário entender a relação entre idades da matriz de corte e armazenamento dos ovos, para esclarecer os efeitos subsequentes sobre a perda de água dos ovos férteis durante o transporte. A eclodibilidade e a qualidade dos pintos são influenciadas pelo tempo de exposição a condições microclimáticas adversas. Entretanto, existem poucas informações científicas relacionadas ao transporte de ovos férteis e sua interação com a idade de matrizes e condições de armazenamento nos incubatórios e matrize iros de corte.

Em função disso, objetivou-se, por meio desta pesquisa, avaliar a perda de água de ovos férteis oriundos de diferentes idades de matrizes de corte e tempo de estocagem durante o transporte.

\section{MATERIAL E MÉTODOS}

A pesquisa foi conduzida nas instalações do matrizeiro e do incubatório avícola de uma empresa integradora, no Estado de São Paulo. Os períodos experimentais foram de novembro a dezembro de 2010 e de fevereiro a abril de 2011.

Para o transporte de ovos férteis, foi realizado um perfil térmico do caminhão de transporte tipo contêiner, modelo Constellation 26-370 6 × 4 tractor, da Volkswagen, apresentando as dimensões de 7,5 metros de comprimento, 2,6 metros de largura e 2,6 metros de altura, com três eixos. A calibragem dos pneus foi de 100 libras, sendo o tipo de suspensão com molas parabólicas e amortecedores hidráulicos de dupla ação. O equipamento de climatização era regulado para as condições de temperatura de $24{ }^{\circ} \mathrm{C}$ e umidade relativa de $80 \%$.

A capacidade total de carregamento do caminhão era de aproximadamente 592 caixas de ovos empilhadas e distribuídas na área da carroceria. Porém os carregamentos estudados apresentaram diferentes densidades de caixas de ovos (302 caixas $=72.480$ ovos na primavera, 362 caixas $=$ 86.644 ovos no verão e 467 caixas $=112.080$ ovos no outono). As caixas foram colocadas em pilhas ao longo do caminhão, e todos os carregamentos estudados tiveram de seguir a logística de transporte e demanda da empresa, não podendo ser alterado (Figura 1). Para as análises de perda de 
água dos ovos, foram utilizadas seis caixas (1.440 ovos) para cada carregamento ou viagem, totalizando 18 caixas de ovos (4.320 ovos), as quais foram localizadas no centro da carroceria do caminhão, devido ser uma região que apresentou menor heterogeneidade térmica, conforme os autores NAZARENO et al. (2013).

As caixas de transporte de ovos possuíam as dimensões externas de $63 \mathrm{~cm}$ de comprimento, $32,5 \mathrm{~cm}$ de largura e $30 \mathrm{~cm}$ de altura, e espessura de $3 \mathrm{~mm}$, com aberturas nas laterais de $3,2 \mathrm{~cm} \mathrm{x}$ $1,1 \mathrm{~cm}$ e no fundo de $2 \times 2 \mathrm{~cm}$. Cada caixa suportava 240 ovos, que equivalem a 8 bandejas de plástico com as dimensões de 30 x $30 \mathrm{~cm}$, com capacid ade de 30 ovos.
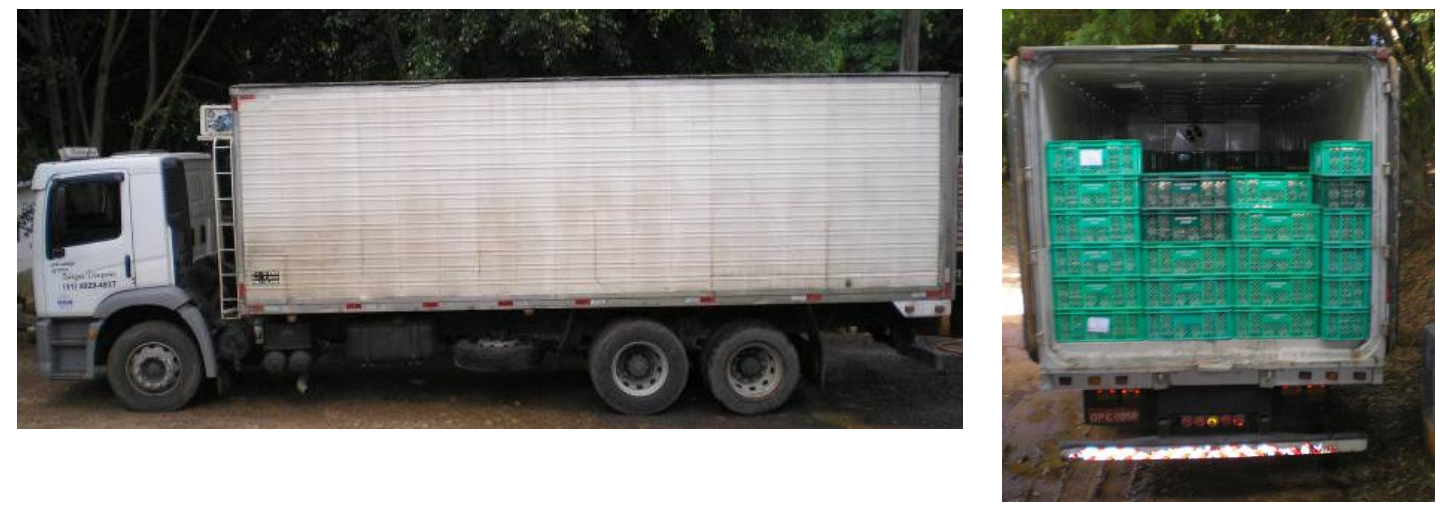

FIGURA 1. Caminhão de transporte climatizado carregado de ovos férteis utilizado na pesquisa. Acclimatized transport trucks loaded with fe rtile eggs used in this study.

Foram realizados os monitoramentos de três viagens, cada uma com um percurso de $60 \mathrm{~km}$. A cada estação do ano (primavera, verão e outono), foi realizada uma viagem. A caracterização do ambiente térmico do contêiner climatizado foi realizada por meio de registros a cada minuto, das seguintes variáveis microclimáticas: temperatura $\left(\mathrm{T} ;{ }^{\circ} \mathrm{C}\right)$ e umidade relativa do ar (UR; \%). A aquisição desses dados foi realizada por meio de 18 dataloggers da marca Logen, em que foi colocado um datalogger no interior das caixas, sendo que foi utilizado um datalogger em cada caixa de estudo, totalização seis dataloggers por viagem, e o restante dos 12 dataloggers foram distribuídos ao longo do perfil da carroceria do caminhão. Os valores de temperatura, umidade relativa e pressão atmosférica média para Amparo-SP, foram utilizados para o cálculo da entalpia específica (h; $\mathrm{kJ} \mathrm{kg}^{-1}$ ar seco) de acordo com a equação de RODRIGUES et al. (2011). Essa grandeza psicrométrica expressa a quantidade de calor existente no ambiente interno do contêiner.

Com relação à massa dos ovos férteis $(\mathrm{kg})$ durante o transporte, foram selecionadas três diferentes idades de matrizes de corte, da linhagem Cobb Fast, provenientes do mesmo lote, e analisadas ao longo do tempo, para avaliar a influência das cond ições microclimáticas do transporte sobre as idades das matrizes de corte, tempos de estocagem, na perda de água dos ovos férteis.

Por meio de um levantamento bibliográfico, conforme os autores ELIBOL et al. (2002), RIBEIRO et al. (2007), TANURE et al. (2009) e NAZARENO et al. (2014b), foram selecionadas e classificadas três idades de matrizes de corte, para as análises: nova $=29-30$ semanas, intermediária $=42-43$ semanas e velha $=52-53$ semanas.

A coleta dos dados foi realizada separadamente para cada idade das matrizes, ou seja, quando as matrizes atingiam as três idades (nova, intermediária e velha), eram coletados 1.440 ovos, que correspondiam a seis caixas de ovos férteis. Em cada uma dessas caixas de ovos, foi colocado um datalogger, para assegurar a temperatura do ar e aumidade relativa que chegava até os ovos férteis.

Todo o planejamento da pesquisa foi realizado para que os tempos de estocagem encerrassem no mesmo período, para a realização do transporte dos ovos férteis (por meio de um caminhão climatizado) com destino ao incubatório. Ou seja, houve uma organização prévia para a armazenagem: três dias antes do transporte foram armazenados 480 ovos; no segundo dia, mais 480 ovos, e no dia do transporte 480 ovos foram selecionados para a pesquisa. 
Os períodos de estocagem dos ovos no matrizeiro foram contabilizados conforme a logística de transporte da empresa, e essa não admitia que os ovos férteis ficas sem armazenados por muitas horas no matrizeiro, devido à dimensão da sala e ao tamanho da produção (ovos/dia), pois essas limitações poderiam comprometer o sistema de climatização da sala de ovos. Os intervalos de tempo de estocagem foram representados por: $\mathrm{T} 1=50-52 \mathrm{~h} ; \mathrm{T} 2=26-30 \mathrm{~h}$, e $\mathrm{T} 3=3-6 \mathrm{~h}$, respectivamente. As caixas de ovos dos tratamentos foram localizadas num ponto central da sala.

Adotou-se o parâmetro massa do ovo como variável resposta, pois o mesmo é um fator de seleção no processo, considerado pela indústria avícola. Foram avaliadas as massas de 140 ovos individualizados de cada tratamento (idade de matrizes e os tempos de estocagem), durante a entrada e a saída dos mesmos na carroceria do caminhão transportador, com intuito de identificar a perda de água dos ovos durante o percurso (matrizeiro ao incubatório), conforme teste prévio e metodologia descrita pelos autores NAZARENO et al. (2014b), verificando-se que 140 ovos por tratamento seria o ideal para determinar a perda de água dos ovos. O equipamento utilizado para aferir a massa $(\mathrm{kg})$ dos ovos foi uma balança semianalítica da marca BEL equipamentos Ltda., modelo THB 600, com escala e precisão de $0,1 \mathrm{~g}$.

Os dados de perda de água dos ovos foram transformados utilizando o Logit da perda de água. Essa transformação de dados é necessária para atender aos pressupostos de uso do modelo de regressão, que traz a perda (que varia de 0 a 100\%) para escala de $-\infty$ a $+\infty$. O Logit da perda de água dos ovos foi calculada pela eq. (1) descrita abaixo:

$$
\operatorname{LOGIT}_{\text {PERDA }}=\log \left(\frac{\mathrm{P}}{1-\mathrm{P}}\right)
$$

em que,

LOGIT_PERDA - logaritmo da perda de água dos ovos férteis;

$\mathrm{P}$ - perda de água dos ovos (\%).

O inverso do logaritmo da perda de água dos ovos foi utilizado para calcular o valor da perda de água em percentual, descrita na eq. (2) a seguir:

$$
\mathbf{P}=\frac{\mathbf{e}^{\text {LOGITPERA }_{\text {PER }}}}{\left(1+\mathbf{e}^{\text {LOGITPERDA })}\right.}
$$

Para a avaliação de perda de água dos ovos férteis, o delineamento experimental consistiu em uma estrutura de parcelas do tipo inteiramente aleatorizada. A estrutura de tratamento foi o esquema fatorial 3 x 3, que representou as três idades de matrizes e os três tempos de estocagem, com 140 repetições.

Com relação à avaliação da influência da idade das matrizes e os tempos de estocagem na perda de água dos ovos férteis, em relação às condições ambientais no transporte, utilizou-se da análise estatística da classe de modelos lineares mistos (VERBEKE \& MOLENBERGHS, 2000; VIEIRA, 2008).

Considerando que as variáveis aleatórias massa do ovo e microclimáticas foram modeladas pela distribuição normal, o modelo estatístico pode ser descrito pela eq. (3)

$$
\begin{aligned}
\mathbf{Y}_{i} & =\mathbf{X}_{i} \beta+\mathbf{Z}_{i} \mathbf{b}_{i}+\varepsilon_{i} \\
\mathbf{b}_{i} & \sim N(\mathbf{0}, \mathbf{D}) \\
\varepsilon_{i} & \sim N\left(\mathbf{0}, \Sigma_{i}\right)
\end{aligned}
$$


em que,

$\mathbf{X}_{i}$ e $\mathbf{Z}_{i}$ as matrizes de delineamento (contendo fatores e/ou covariáveis bioclimáticas) para o vetor $p$-dimensional $\beta$, que contém os efeitos fixos e o vetor $q$-dimensional $\mathbf{b}_{i}$ dos efeitos aleatórios para modelar o efeito das aves, que possui distribuição normal multivariada com média $\mathbf{0}$ e matriz de covariâncias $\mathbf{D}$, e

$\varepsilon_{i}$ é o vetor de erros aleatórios com distribuição normal multivariada com média $\mathbf{0}$ e matriz de covariância $\Sigma_{i}$. Assume-se que os efeitos aleatórios associados às aves são independentes dos erros $\varepsilon_{i}$.

$\mathrm{Na}$ análise estatística da carroceria climatizada, foi utilizado o modelo linear misto assumindo uma estrutura de covariância autorregressiva de primeira ordem. As variáveis respostas do modelo foram temperatura, umidade relativa e entalpia específica, em função do fator estação do ano (primavera, verão e outono). Vale ressaltar que cada estação representou uma idade de matriz (primavera $=$ nova; verão $=$ intermediária, e outono $=$ velha). Os resultados foram submetidos ao teste $\mathrm{F}$ para comparação entre as médias. 2010).

Todas as análises estatísticas foram realizadas por meio do software estatístico SAS (SAS,

\section{RESULTADOS E DISCUSSÃO}

Avaliando-se as condições microclimáticas de transporte de ovos, verificou-se diferença entre as estações $(\mathrm{P}<0,05)$, para todas as variáveis microclimáticas, sendo que durante o verão foram registrados os maiores valores médios (Tabela 1).

TABELA 1. Valores médios do microclima do caminhão em diferentes estações do ano. Average values of microclimate truck in different seasons.

\begin{tabular}{cccc}
\hline \multirow{2}{*}{ Estações do ano } & $\begin{array}{c}\text { Temperatura } \\
\left({ }^{\circ} \mathrm{C}\right)\end{array}$ & $\begin{array}{c}\text { Umidade relativa } \\
(\%)\end{array}$ & $\begin{array}{c}\text { Entalpia específica } \\
\left(\mathrm{kJ} \mathrm{kg}^{-1} \text { de ar seco }\right)\end{array}$ \\
\hline Primavera & $24,2 \mathrm{~B}$ & $71,5 \mathrm{C}$ & $59,0 \mathrm{~B}$ \\
Verão & $26,0 \mathrm{~A}$ & $91,0 \mathrm{~A}$ & $74,0 \mathrm{~A}$ \\
Outono & $22,5 \mathrm{C}$ & $81,5 \mathrm{~B}$ & $57,4 \mathrm{C}$ \\
\hline Teste $\mathrm{F}$ & $42,43^{*}$ & $18,34^{*}$ & $63,02^{*}$ \\
\hline
\end{tabular}

Médias com letras diferentes nas colunas, diferem entre si, a $5 \%$ de probabilidade, pelo teste $\mathrm{F} .{ }^{*}$ - significativo a $5 \%$ de probabilidade $(\mathrm{P}<0,05)$.

De modo geral, as fases de verão e primavera ofereceram as piores condições térmicas durante o transporte de ovos férteis, apresentando os valores médios de temperatura entre 24,2 e $26,0{ }^{\circ} \mathrm{C}$.

Ao observar a temperatura média da carroceria do caminhão, nota-se que a mesma esteve acima das faixas ideais $\left(10\right.$ a $\left.20^{\circ} \mathrm{C}\right)$ para todas as estações do ano, conforme as condições indicadas (FASENKO et al., 1991; AK YUREK \& OKUR, 2009; SALAHI et al., 2012; NAZARENO et al., 2013). O valor médio de temperatura mais próximo do ideal ocorreu no outono, com $22,5^{\circ} \mathrm{C}$. Isso demonstra a necessidade de maior controle do sistema de climatização do caminhão durante a primavera e o verão. Resultados similares foram encontrados por DAMRON et al. (1994), GAST \& HOLT (2000) e ANDERSON et al. (2008), ao avaliarem o transporte de ovos comerciais. Os mesmos autores observaram que as estações do ano exercem forte influência sobre a variação da temperatura no contêiner climatizado, sendo que o verão foi considerado a pior época para se transportar ovos. Quando os ovos férteis são submetidos a altas temperaturas fora da incubadora, pode ficar comprometida a viabilidade embrionária nos estádios iniciais de desenvolvimento, 
reduzir a taxa de eclosão, além de aumentar a taxa de mortalidade (CONY et al., 2008; REIJRINK et al., 2010; BARACHO et al., 2013).

Com relação à variação da umidade relativa, verifica-se que os maiores valores médios foram registrados no verão e outono, em torno de 81,5 e $90 \%$. Esses valores de umidade relativa podem ser justificados pela elevada densidade de caixas no caminhão (86.644 e 112.080 ovos férteis) e com isto maiores perdas de água dos ovos férteis durante o transporte (Tabela 1). Vale lembrar que, quando a umidade relativa está acima da faixa ideal $(55$ - 75\%), tem-se o início da condensação sobre os ovos, que resultará em um ambiente mais propício para o crescimento de microrganismos (fungos e bactérias) nos ovos férteis (MOURA et al., 2008; PLETI et al., 2009; BARACHO et al., 2010; FREITAS et al., 2011; BARACHO et al., 2013). O período da primavera foi o que apresentou menor valor médio de umidade relativa, o qual foi registrado em 71,5\%. Esse foi o único período que, esteve dentro das faixas ideais (55 a 75\%), conforme os autores FASENKO et al. (1991), AKYUREK \& OKUR (2009), SALAHI et al. (2012) e NAZARENO et al. (2013). Essa menor umidade relativa nessa primavera pode ter sido influenciada pela estação do ano e pela menor quantidade de ovos (72.480) transportada, a qual proporcionou menor perda de umidade dos ovos férteis.

Os maiores valores médios de entalpia específica da carroceria climatizada foram verificados

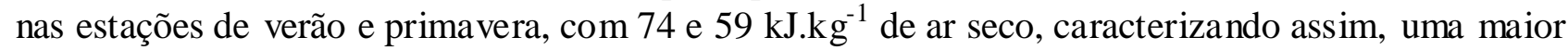
quantidade de calor existente no ambiente interno da carroceria climatizada. Esses valores podem ser atribuídos à elevação da temperatura em conjunto com a umid ade relativa.

Houve interação entre idade de matrizes e os intervalos de tempo de estocagem $(\mathrm{P}<0,05)$ quanto à perda de água dos ovos férteis durante o transporte (Tabela 2). No entanto, a diferença da perda de água dos ovos durante o transporte foi pequena para todas as idades de matrizes e os tempos de estocagem.

TABELA 2. Perda de água dos ovos férteis em função das idades de matrizes e tempos de estocagem, durante o transporte dos ovos férteis. Water loss of fertile eggs depending on the age of breeders and storage time, during the transport of fertile eggs.

\begin{tabular}{l|ccc}
\hline \multirow{2}{*}{ Idade das matrizes de corte } & \multicolumn{3}{c}{ Perda de água dos ovos férteis (\%) } \\
\cline { 2 - 4 } & \multicolumn{3}{|c}{ Tempos de estocagem $(\mathrm{h})$} \\
\cline { 2 - 4 } & $03-06$ & $26-30$ & $50-52$ \\
\hline Nova (29-30 semanas; Primavera) & $0,07 \mathrm{Ba}$ & $0,06 \mathrm{Ca}$ & $0,08 \mathrm{Ca}$ \\
Intermediária (42-43 semanas; Verão) & $0,13 \mathrm{Ac}$ & $0,16 \mathrm{Ab}$ & $0,19 \mathrm{Aa}$ \\
Velha (52-53 semanas; Outono) & $0,09 \mathrm{Bb}$ & $0,08 \mathrm{Bb}$ & $0,12 \mathrm{Ba}$ \\
\hline Teste F & $16,41^{*}$ &
\end{tabular}

Médias com letras diferentes, maiúsculas nas colunas e minúsculas nas linhas, diferem entre si, a 5\% de probabilidade, pelo teste $\mathrm{F}$. * - significativo a $5 \%$ de probabilidade $(\mathrm{P}<0,05)$.

Os maiores valores de perda de água dos ovos foram verificados para as matrizes com idade intermediárias, sendo as proporções para os três períodos de estocagem de 0,13;0,16 e 0,19\%, respectivamente.

Com relação ao tempo de estocagem, as maiores médias de perdas de água dos ovos ocorreram no período de 50-52 h, para todas as idades de matrizes. Quanto maior o tempo de estocagem, maior será a perda de água dos ovos SCATOLINI-SILVA et al. (2013) e ALSOBAYEL et al. (2013), independentemente do trajeto percorrido. Portanto, o maior valor médio de perda de água dos ovos férteis foi detectado na idade de matriz intermediária e no tempo de estocagem de 50-52 h, com 0,19\%. FERREIRA et al. (2006) obtiveram resultado semelhantes a este estudo para o tempo de estocagem dos ovos férteis ( 8 dias de armazenamento); porém, com relação a idade das matrizes, esses autores evidenciaram a maior perda de massa dos ovos nas matrizes de idade velha, discordando dos encontrados nesta pesquisa. Também, segundo os autores ZAKARIA et al. (2009) 
e ALSOBAYEL et al. (2013), ovos oriundos de matrizes de idade velha e o maior tempo de estocagem perdem mais massa em relação aos demais (ovos oriundos de matrizes novas e menor tempo de armazenamento); porém, nenhum desses autores avaliou as condições térmicas onde esses ovos foram estocados. Sendo assim, pode-se dizer que, quando esses ovos estão sob condições térmicas diferentes, essa perda de água pode ser alterada, como foi o caso dos o vos oriundos de matrizes intermediárias do presente estudo. Então, por meio destes resultados, verificou-se que as diferentes estações do ano e a densidade da carga podem influenciar de forma adversa no microclima da carga de ovos férteis transportada.

As maiores médias de perda de água dos ovos foram observadas para a idade de matriz intermediária para todos os tempos de estocagem (Figura 2). Tal variação pode ser explicada pelo transporte durante o verão $\left(26^{\circ} \mathrm{C} ; 91 \%\right)$, quando foram registradas as piores condições térmicas, sendo a maior variação de temperatura e umidade relativa, e com o maior carregamento (112.080 unid ades de ovos).

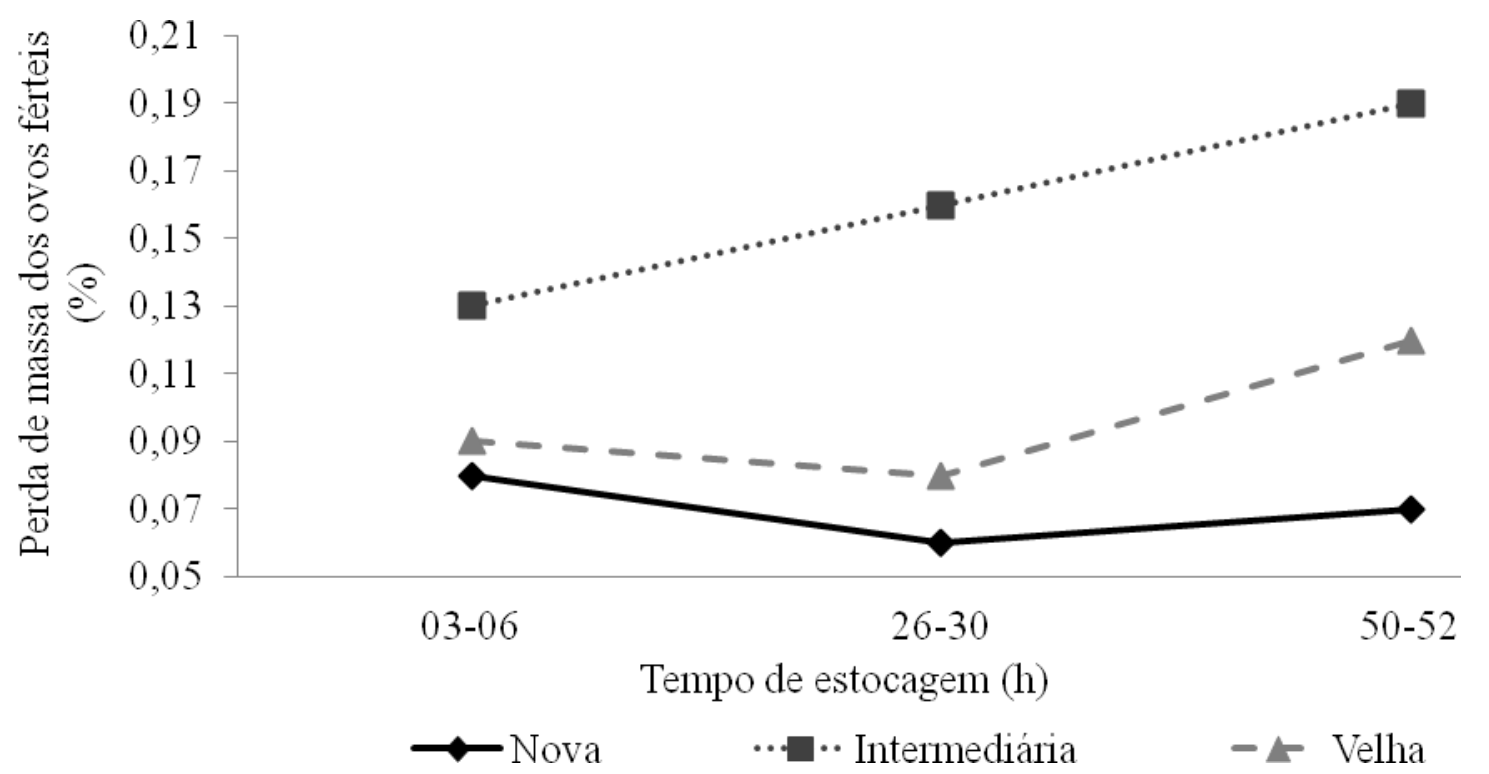

FIGURA 2. Perda de água dos ovos férteis em função das idades das matrizes de corte e dos tempos de estocagem, durante o transporte dos ovos férteis. Water loss of fertile eggs depending on the age of broiler breeders and storage time, during the transport of fertile eggs.

Sabe-se que a idade da matriz influencia na perda de massa dos ovos férteis (TANURE et al., 2009; AKYUREK \& OKUR, 2009; CORRÊA et al., 2011; SANTANA et al., 2013). Porém, quando esses ovos estão submetidos às condições microclimáticas de transporte adversas e densidades de caixas (ovos férteis) diferentes, as perdas de água dos ovos podem aumentar, independentemente da idade da matriz oriunda, como pode ser verificado na Figura 2.

Diante disso, pode-se dizer que os fatores que influenciaram na perda de água dos ovos durante o transporte da carga foram a condição microclimática, em função das estações do ano, e a densidade da carga. Por isso, é muito importante que exista uma uniformização da carga de ovos na carroceria do caminhão, fazendo um manejo da carga conforme a estação do ano (diminuindo ou aumentando a densidade de caixas), ou seja, nas estações quentes (verão) diminuir a densidade das caixas, e nas frias (inverno) aumentar a densidade das caixas.

Observou-se que a variabilidade da temperatura (desvio-padrão da temperatura) apresentou uma grande influência no logit da perda de água dos ovos com 0,52 unidades, o que equivale a uma perda de 0,6\% (Tabela 3). Este resultado sugere que a oscilação da temperatura no baú climatizado pode ser mais prejudicial à perda de água dos ovos férteis do que a ele vação da temperatura. 
Ao aumentar $1 \%$ de umidade relativa no logit da perda de água dos ovos, houve a diminuição de $-0,009$ unidade no logit, o que equivale a $0,5 \%$ de perda de água dos ovos. A variabilidade da umidade relativa (desvio-padrão da umidade relativa) apresentou o mesmo comportamento do logit da perda de água da umidade relativa. Portanto, pode-se dizer que a umidade relativa e a amplitude dela apresentaram o mesmo percentual na perda de água dos ovos. Deste modo, é muito importante o controle das condições microclimáticas dentro do baú climatizado, durante o transporte dos ovos, para evitar as perdas de água excessivas, sem causar danos ao embrião.

TABELA 3. Simulação das variações de temperatura e umidade relativa no modelo Logit_perda em função da perda de água dos ovos férteis. Simulation of changes in tempe rature and relative humidity in Logit_loss model due to loss of water from the fertile eggs.

\begin{tabular}{ccc}
\hline Variação da temperatura $\left({ }^{\circ} \mathrm{C}\right)$ & Umidade relativa $(\%)$ & Perda de água dos ovos $(\%)$ \\
\hline 0,52 & - & 0,60 \\
- & 1,0 & 0,50 \\
- & 1,5 & 0,51 \\
- & 2,0 & 0,52 \\
- & $\Delta \mathrm{UR}$ & 0,50 \\
\hline
\end{tabular}

\section{CONCLUSÕES}

Independentemente da idade da matriz e da condição do microclima, o aumento do tempo de estocagem leva a maior perda de água, assim como a piores condições microclimáticas (alta umidade relativa e temperatura do ar).

A oscilação da temperatura do ar aumentou muito mais a perda de água dos ovos no baú climatizado, do que o valor da temperatura em si. Para cada variação de $1 \%$ de aumento na umidade relativa, aumenta-se $0,5 \%$ na perda de água dos ovos férteis.

\section{AGRADECIMENTOS}

Os autores agradecem à Fundação de Amparo à Pesquisa do Estado de São Paulo (FAPESP), pelo apoio financeiro a esta pesquisa; ao CNPq e CAPES, pela concessão da bolsa de estudo.

\section{REFERÊNCIAS}

AKYUREK, H., OKUR, A. A. Effect of storage time, temperature and hen age on egg quality in free range layer hens. Journal of Animal and Veterinary Advances, v. 8, n. 10, p. 1953-1958, ago./set. 2009. Disponível em: <http://docsdrive.com/pdfs/medwelljournals/javaa/2009/19531958.pdf>. Acesso em 17 dez. 2014.

ALSOBAYEL, A. A.; ALMARSHADE, M. A.; ALBADRY, M. A. Effect of breed, age and storage period on egg weight, egg weight loss and chick weight of commercial broiler breeders raised in Saudi Arabia. Journal of the Saudi Society of Agricultural Sciences, Ryadh, v. 12, n. 1, p. 53-57, 2013. Disponível em: <http //ac.els-cdn.com/S1658077X12000252/1-s2.0-

S1658077X12000252-main.pdf?_tid=09825648-85e2-11e4-b77600000aab0f6c\&acdnat=1418816839_03b01a819a8e9ff6575c1b9d7b315c97>. Doi: 10.1016/j.jssas.2012.06.003

ANDERSON, K. E.; PATTERSON, P. H.; KOELKEBECK, K. W.; DARRE, M. J.; CAREY, J. B.; AHN, D. U.; ERNST, R. A.; KUNEY, D. R.; JONES, D. R. Temperature sequence of eggs from oviposition through distribution: transportation - part 3. Poultry Science, Savoy, v. 87, n. 6, p. 1195-1201, 2008. Disponível em:

<http://ps.oxfordjournals.org/content/87/6/1195.full.pdf+html?sid=62bfa2e1-689a-414a-b43696c3352bc6d0>. Doi: 10.3382/ps.2007-00236.

BARACHO, M. S.; NÄÄS, I. A.; GIGLI, A. C. S. Impacto das variáveis ambientais em incubatório de estágio múltiplo de frangos de corte. Engenharia Agrícola, Jaboticabal, v. 30, n. 4, p. 563-577, 
2010. Disponível em: <http://www.scielo.br/scielo.php?pid=S0100-

69162010000400001\&script=sci_arttext $>$. Doi: $10.1590 /$ S0100-69162010000400001

BARACHO, M. S.; NÄ̈̈S, I. A.; NEVES, D. P.; JULIANO DE A. CASSIANO, J. A.; LIMA, F. G.; MOURA, D. J.; GARCIA, R. G. Estimating the most important criteria for hatching eggs as function of broiler breeders age. Engenharia Agrícola, Jaboticabal, v. 33, n. 3, p. 445-452, 2013. Disponível em: <http://www.scielo.br/scielo.php?script=sci_arttext\&pid=S0100-

69162013000300002>. D oi:10.1590/S0100-69162013000300002

CONY, H. C.; VIEIRA, S. L; BERRES, J.; GOMES, H. A.; CONEGLIAN, J. L. B.; FREITAS, D. M.. Técnicas de pulverização e imersão com distintos desinfetantes sobre ovos incubáveis. Ciência Rural, Santa Maria, v. 38, n. 5, p. 1407-1412. 2008. Disponível em:

<http://www.scielo.br/scielo.php?pid=S0103-84782008000500033\&script=sci_arttext $>$. Doi: 10.1590/S0103-84782008000500033

CORRÊA, A. B.; SILVA, M. A.; CORRÊA, G. S. S.; SANTOS, G. G.; FELIPE, V. P. S.; WENCESLAU, R. R.; SOUZA, G. H.; CAMPOS, N. C. F. L. Efeito da interação idade da matriz X peso do ovo sobre o desempenho de codornas de corte. Arquivo B rasileiro de Medicina Veterinária e Zootecnia, Belo Horizonte, v. 63, n. 2, p. 433-440, 2011. Disponível em: $<$ http://www.scielo.br/scielo.php?pid=S0102-09352011000200022\&script=sci_arttext $>$. Doi: 10.1590/S0102-09352011000200022

DAMRON, B. L., DOUGLAS, C. R.; JACOBS, R. D. Temperature patterns in commercial egg transport vehicles. Journal of Applied Poultry Research, Athens, v. 3, n. 2, p. 193-198, 1994. Disponível em: <http://japr.oxfordjournals.org/content/3/2/193.full.pdf+html>. Doi: 10.1093/japr/3.2.193

ELIBOL, O.; PEAK, S. D.; BRAKE, J. Effect of flock age, length of egg storage, and frequency of turning during storage on hatchability of broiler hatching eggs. Poultry Science, Savoy, v.81, p.945-950, 2002. Disponível em: <http://ps.oxfordjournals.org/content/81/7/945.full.pdf>. Doi: $10.1093 / \mathrm{ps} / 81.7 .945$

FASENKO, G. M.; ROBINSON, F. E.; ARMSTRONG, J. G.; CHURCH, J. S.; HARDIN, R. T.; PETITTE, J. N. Variability in pre-incubation embryo development in domestic fowl: effects of nest holding time and method of egg storage. Poultry Science, Savoy, v. 70, n. 9, p. 1876-1881, 1991. Disponível em: <http://ps.oxfordjournals.org/content/70/9/1876.full.pdf>. Doi:10.3382/ps.0701876 FERREIRA, F. C.; LARA, L. J. C.; BAIÃO, N. C.; LANA, A. M. Q.; LÓPEZ, C. A. A.; MORAES, D. T. Influência da idade da matriz e do período de armazenamento dos ovos sobre a uniformidade dos pintos aos 11 dias de idade. Revista Brasileira de Ciência Avícola, Campinas, supl. 8, p.17, maio/ago. 2006.

FREITAS, L. W.; PAZ, I. C. L. A.; GARCIA, R. G.; CALDARA, F. R.; SENO, L. O.; FELIX, G. A.; LIMA, N. D. S.; FERREIRA, V. M. O. S.; CAVICHIOLO, F. Aspectos qualitativos de ovos comerciais submetidos a diferentes condições de armazenamento. Revista Agrarian, Dourados, v. 4, n. 11, p. 66-72, abr./mai. 2011. Disponível em: <http:

file:///C:/Documents\%20and\%20Settings/x- user/Meus\%20documentos/Download s/998-2912-1PB.pdf>. Acesso em: $14 \mathrm{dez} 2014$.

GAST, R. K.; HOLT, P. S. Influence of the level and location of contamination on the multiplication of Salmonella enteritidis at different storage temperatures in experimentally inoculated eggs. Poultry Science, Savoy, v. 79, n. 4, p. 559-563, 2000. Disponível em: $<$ http://ps.oxford journals.org/content/79/4/559.full.pdf+html?sid=66683896-7a94-4024-8a2753fd87838d21>. doi:10.1093/ps/79.4.559

MADEDDU, M.; ZANIBONI, L.; MANGIAGALLI, M. G; CASSINELLI, C.; CEROLINI, S. Egg related parameters affecting fertility and hatchability in the Italian bantam breed Mericanel della Brianza. Animal Reproduction Science, Amsterdam, v. 137, n. 3-4, p. 214- 219, 2013. Disponível em: <http://ac.els-cdn.com/S0378432013000031/1-s2.0-S0378432013000031- 
main.pdf?_tid=e27bce28-85df-11e4-bc56-

00000aab0f01\&acd nat=1418815915_2c85829c14c1778b09cfcd057336bb31>. Doi: 10.1016 / j.anireprosci.2013.01.002

MOURA, A. M. A.; OLIVEIRA, N. T. E.; THIEBAUT, J. T. L.; MELO, T. V. Efeito da temperatura de estocagem e do tipo de embalagem sobre a qualidade interna de ovos de codornas japonesas (Coturnix japonica). Ciência e Agrotecnologia, Lavras, v. 32, n. 2, p. 578-583, 2008. Disponível em: <http://www.scielo.br/scielo.php?script=sci_arttext\&pid=S141370542008000200036>. Doi: 10.1590/S1413-70542008000200036

NAZARENO, A. C.; SILVA, I. J. O; VIEIRA, A. M. C.; VIEIRA, F. M. C.; MIRANDA-SILVA, K. O. Transporte de ovos férteis: Influência das idades das matrizes, tempos de estocagem e das estradas. Revista Brasileira de Engenharia Agrícola e Ambiental, Campinas Grande, v. 18, n. 3, p. 338-343, 2014a. Disponível em:

<http://www.scielo.br/scielo.php?script=sci_arttext\&pid=S1415-43662014000300014>. Doi: 10.1590/S1415-43662014000300014

NAZARENO, A. C.; SILVA, I. J. O.; VIEIRA, A. M. C.; VIEIRA, F. M. C. Microclima, idade das matrizes e tempo de estocagem influenciando nas respostas produtivas de ovos férteis. Revista Brasileira de Engenharia Agrícola e Ambiental, Campinas Grande, v. 18, n. 11, p. 1172-1178, 2014b. Disponível em: <http://www.scielo.br/scielo.php?pid=S141543662014001100012\&script=sci_arttext $>$. Doi: 10.1590/1807-1929/agriambi.v18n11p1172-1178

NAZARENO, A. C.; SILVA, I. J. O.; VIEIRA, F. M. C.; CAMARGO, J. R.; MEDEIROS, S. R. R. Caracterização do microclima dos diferentes layouts de caixas no transporte de ovos férteis.

Revista Brasileira de Engenharia Agrícola e Ambiental, Campinas Grande, v. 17, n. 3, p. 327 332, 2013. Disponível em: <http://www.scielo.br/scielo.php?script=sci_arttext\&pid=S141543662013000300012>. Doi: 10.1590/S1415-43662013000300012

OVIEDO-RONDÓN, E. O.; WINELAND, M. J.; SMALL, J.; CUTCHIN, H.; MCELROY, A.; BARRI, A.; MARTIN, S. Effect of incubation temperatures and chick transportation conditions on bone development and leg health. Journal of Applied Poultry Research, Newtown, v. 18, n. 4, p. 671- 678. 2009. Disponível em:

<http://japr.oxfordjournals.org/content/18/4/671.full.pdf?origin=publication_detail >. Doi: 10.3382/japr.2008-00135

PLETI, A. K.; LIMA, J. J.; CANDIDO, L. M. B. Qualidade interna do ovo de avestruz após estocagem em temperatura ambiente e refrigerada. Ciência Rural, Santa Maria, v. 39, n. 6, 2009. Disponível em: <http://www.scielo.br/scielo.php?script=sci_arttext\&pid=S010384782009000600035>. Doi: 10.1590/S0103-84782009005000113

REIJRINK, I. A. M.; BERGHMANS, D.; MEIJERHOF, R.; KEMP, B.; VAN DEN BRAND, H. Influence of egg storage time and preincubation warming profile on embryonic development, hatchability, and chick quality. Poultry Science, Savoy, v. 89, n. 6, p. 1225-1238, 2010. Disponível em: <http://ps.oxfordjournals.org/content/88/12/2649.full.pdf+html>. Doi: 10.3382/ps.2008-00523

RIBEIRO, B. R. C.; LARA, L. J. C.; BAIÃO, N. C.; LOPEZ, C. A. A.; FIUZA, M. A.; CANÇADO, S. V.; SILVA, G. M. M. Efeito do nível de ácido linoléico na ração de matrizes pesadas sobre o peso, composição e eclosão dos ovos. Arquivo B rasileiro de Medicina Veterinária e Zootecnia, Belo Horizonte, v.59, p.789-796, 2007. Disponível em: <http://www.scielo.br/scielo.php?pid=S0102-09352007000300034\&script=sci_arttext>. Doi: 10.1590/S0102-09352007000300034

RODRIGUES, V. C.; SILVA, I. J. O.; VIEIRA, F. M. C.; NASCIMENTO, S. T. A correct enthalpy relationship as thermal comfort index for livestock. International Journal of Biometeorology, Heidelberg, v. 55, n. 3, p. 455-459, 2011. Disponível em: $<$ http://download. springer.com/static/pdf/828/art\%253A10.1007\%252Fs00484-010-0344- 
y.pdf?auth66=1418815339_f00e8892de91ee4b613eaa762a3bc3a1\&ext=.pdf >. Doi: 10.1007/s00484-010-0344-y

SALAHI, A.; KHABISI, M. M.; PAKDEL, A.; BAGHBANZADEH, A. Effects of cold stress during transportation on hatchability and chick quality of broiler breeder eggs. Turkish Journal of Veterinary \& Animal Sciences, Ankara, v. 36, n. 2, p. 159-167, 2012. Disponível em: $<$ http://www.researchgate.net/publication/221705182_Effects_of_cold_stress_during_transportatio n_on_hatchability_and_chick_quality_of_broiler_breeder_eggs>. Doi:10.3906/vet-1101-750

SANTANA, M. H. M.; GIVISIEZ, P. E. N.; FIGUEIREDO JÚNIOR, J. P.; SANTOS, E. G. Avaliação de protótipos de incubadoras sobre os parâmetros embrionários de ovos férteis caipiras. Revista de Ciências Agrárias, Lisboa, v. 36, n. 2, p. 157-162, abr. 2013. Disponível em: <http://www.scielo.oces.mctes.pt/scielo.php?pid=S0871018X2013000200004\&script=sci_arttext\&tlng=pt>. Acesso em: 14 dez. 2014.

SAS Institute. Statistical analysis system: Realease 9.2, (software). Cary, 2010. 620p.

SCATOLINI-SILVA, A. M.; BORBA, H. A.; GIAMPIETRO-GANECO, A. B.; SOUZA, P. A. C.; BOIAGO, M. M.; MELLO, J. L. M. D.; VAZ, A. B. S. Qualidade física de ovos armazenados em diferentes condições de embalagens sob temperatura ambiente. Archivos de Zootecnia, Córdoba, v. 62 , n. 238, p. 247-254, 2013. Disponivel em: <http://scielo.isciii.es/scielo.php?pid=S000405922013000200010\&script=sci_arttext $>$. Doi: 10.4321/S0004-05922013000200010

TANURE, C. B. G. S.; CAFÉ, M. B.; LEANDRO, N. S. M. ; BAIÃO, N. C.; TRINGHINI, J. H.; GOMES, N. A. Efeitos da idade da matriz leve e do período de armazenamento de ovos incubáveis no rendimento de incubação. Arquivo B rasileiro de Medicina Veterinária e Zootecnia, Belo Horizonte, v. 61, n. 6, p. 1391-1396, 2009. Disponível em:

<http://www.scielo.br/scielo.php?script=sci_arttext\&pid=S0102-09352009000600019>. Doi: 10.1590/S0102-09352009000600019

VERBEKE, G.; MOLENBERGHS, G. Linear mixed models for longitudinal data. New York: Springer Science + Bussiness Media, 2000. 568p.

VIEIRA, A. M. C. Modelagem simultânea de média e dispersão e aplicações na pesquisa agronômica. 2008. 176 f. Tese (Tese em Estatística e Experimentação Agronômica) - Escola Superior de Agricultura "Luiz de Queiroz", Universidade de São Paulo, Piracicaba, 2008.

ZAKARIA, A. H., PLUMSTEAD, P. W., ROMERO-SANCHEZ, H., LEKSRISOMPONG, N., BRAKE, J. The effects of oviposition time on egg weight loss during storage and incubation, fertility, and hatchability of broiler hatching eggs. Poultry Science, Savoy, v. 88, n. 2, p. 2712 -2717, 2009. Disponível em:

<http://www.researchgate.net/publication/38080690_The_effects_of_oviposition_time_on_egg_wei ght_loss_during_storage_and_incubation_fertility_and_hatchability_of_broiler_hatching_eggs>

Doi: 10.3382 / ps.2009-00069 